\title{
Identification of the prognostic value of a 2-gene signature of the WNT gene family in UCEC using bioinformatics and real-world data
}

Yuexin $\mathrm{Hu}^{1,2,3}$, Mingjun Zheng ${ }^{1,2,3,4}$, Dandan Zhang ${ }^{1,2,3}$, Rui Gou ${ }^{1,2,3}$, Ouxuan Liu' ${ }^{1,2,3}$, Shuang Wang ${ }^{1,2,3}$ and Bei $\operatorname{Lin}^{1,2,3,5^{*}}$

\begin{abstract}
Background: The WNT gene family plays an important role in the occurrence and development of malignant tumors, but its involvement has not been systematically analyzed in uterine corpus endometrial carcinoma (UCEC). This study aimed to evaluate the prognostic value of the WNT gene family in UCEC.

Methods: Pan-cancer transcriptome data of the UCSC Xena database and Genotype-Tissue Expression (GTEx) normal tissue data were downloaded to analyze the expression and prognosis of 19 WNT family genes in UCEC. A cohort from The Cancer Genome Atlas-Uterine Corpus Endometrial Carcinoma (TCGA-UCEC) was used to analyze the expression of the WNT gene family in different immune subtypes and clinical subgroups. The STRING database was used to analyze the interaction of the WNT gene family and its biological function. Univariate Cox regression analysis and Lasso cox analysis were used to identify the genes associated with significant prognosis and to construct multi signature prognosis model. An immunohistochemical assay was used to verify the predictive ability of the model. Risk score and the related clinical features were used to construct a nomogram.
\end{abstract}

Results: The expression levels of WNT2, WNT3, WNT3A, WNT5A, WNT7A, and WNT10A were significantly different among different immune subtypes and correlated with TP53 mutation. According to the WNT family genes related to the prognosis of UCEC, UCEC was classified into two subtypes $(\mathrm{C1}, \mathrm{C2})$. The prognosis of subtype $\mathrm{C1}$ was significantly better than that of subtype C2. A 2-gene signature (WNT2 and WNT10A) was constructed and the two significantly prognostic groups can be divided based on median Risk score. These results were verified using real-world data, and the nomogram constructed using clinical features and Risk score had good prognostic ability.

Conclusions: The 2-gene signature including WNT2 and WNT10A can be used to predict the prognosis of patients with UCEC, which is important for clinical decision-making and individualized therapy for patients with UCEC.

Keywords: UCEC, WNT2, WNT10A, Prognosis, Nomogram

*Correspondence: linbei88@hotmail.com; blin@cmu.edu.cn

${ }^{5}$ Present Address: 4th Gynecological Ward, Department of Obstetrics and Gynecology, Shengjing Hospital of China Medical University, 36 Sanhao Street, Liaoning 110004 Shenyang, People's Republic of China Full list of author information is available at the end of the article

\section{Background}

Uterine corpus endometrial carcinoma (UCEC) is one of the most common malignancies of the female reproductive system. Epidemiological data have shown that the incidence of UCEC has increased globally in the last two decades [1], and there are expected to be 66,570 new original author(s) and the source, provide a link to the Creative Commons licence, and indicate if changes were made. The images or other third party material in this article are included in the article's Creative Commons licence, unless indicated otherwise in a credit line to the material. If material is not included in the article's Creative Commons licence and your intended use is not permitted by statutory regulation or exceeds the permitted use, you will need to obtain permission directly from the copyright holder. To view a copy of this licence, visit http://creativecommons.org/licenses/by/4.0/. The Creative Commons Public Domain Dedication waiver (http://creativeco mmons.org/publicdomain/zero/1.0/) applies to the data made available in this article, unless otherwise stated in a credit line to the data. 
cases and 12,940 deaths because of UCEC in the United States in 2021 [2]. Because the clinical symptoms of UCEC are predictable, most cases can be diagnosed early, and the 5-year overall survival (OS) rate is more than $90 \%$. However, the prognosis of advanced or recurrent UCEC remains poor, with a 5-year OS rate of less than $30 \%$ for patients at International Federation of Obstetrics and Gynecology (FIGO) stage IV [3]. At present, prognostic predictors for patients with UCEC are primarily based on clinical variables such as age, FIGO stage, and pathological subtypes. Studies have shown that certain genetic or molecular changes can affect UCEC prognosis [4]. In March 2020, National Committee on Computer Network (NCCN) recommended The Cancer Genome Atlas (TCGA) molecular subtype for the first time and included it in the guidelines for diagnosis and treatment of endometrial cancer, heralding the era of genotype based precision therapy. By analyzing the genomic, transcriptome and proteomic characteristics of 373 endometrial cancer patients, TCGA divided endometrial cancer into four subtypes, namely, POLE hyper-mutation, highmutation microsatellite instability (MSI), high-copy number type (such as p53 gene mutation) and non-specific molecular variation (NSMP). TCGA molecular subtype is important in predicting the prognosis of patients with advanced endometrial cancer and in evaluating surgical interventions to preserve reproductive function. However, TCGA molecular subtype is expensive in clinical application and high in medical costs, so we intend to explore a more convenient method to predict endometrial cancer prognosis.

The WNT gene was first cloned from mouse breast cancer induced by mouse papillomavirus, then named Int-1, and was later identified as being homologous to the wingless gene of Drosophila; therefore, these genes are collectively called WNT [5]. At present, 19 kinds of human WNT genes have been discovered, and the secretory glycoprotein encoded by WNT genes is the initiator of the WNT signaling pathway [6]. Numerous studies have demonstrated that abnormal WNT signal activation is involved in the occurrence and development of many kinds of malignant cancers such as gastric cancer [7], breast cancer [8], and colon cancer [9]. However, few studies have been conducted on WNT family genes in UCEC.

The rapid development of high throughput sequencing technology and public databases in recent years provides new ideas for data mining and a better understanding of gene function. For example, the nomogram constructed by Cheng et al. [10] can predict the OS rate of patients with UCEC using immune-related genes. The 4-gene signature constructed with autophagy-related genes based on TCGA by Zhang et al. [11] can be used to predict the prognosis of patients with UCEC. Studies using gene family genes in general to construct risk models in UCEC are relatively rare, and the value of the WNT gene family in the diagnosis and prognosis of UCEC is unclear.

In this study, the prognostic value of the WNT gene family in UCEC was comprehensively analyzed using The Cancer Genome Atlas-Uterine Corpus Endometrial Carcinoma (TCGA-UCEC) data, and the Risk score was constructed. The predictive ability of the Risk score was also validated by using data of 75 UCEC samples from Shengjing Hospital affiliated to China Medical University. The nomogram combined with clinical characteristics provides new insight into personalized prognosis prediction and clinical diagnosis of patients with UCEC.

\section{Materials and methods Data source}

The pan-cancer transcriptome data of 33 cancers from the UCSC Xena database and the transcriptome data of all tissues in the Genotype-Tissue Expression (GTEx) database were downloaded. Limma package [12] was used to analyze the differential expression in TCGA and GTEx datasets. Univariate Cox analysis was used to identify the prognostic WNT gene in pan-cancer. The results of differential expression analysis and prognosis analysis were visualized by pheatmap package.

\section{Clinical relevance of the WNT gene family in UCEC}

UCEC data were downloaded, including the clinical stage, tumor grade, pathological subtypes, TP53 mutation, age, and OS and progression-free survival (PFS) data of patients. The expression of WNT family genes in different immune subtypes and clinical subgroups was analyzed. The interaction of 19 genes was analyzed by the STRING database, the functional enrichment of genes was analyzed by Gene Ontology (GO), and the clusterProfiler package was used for visualization.

\section{Identification of UCEC molecular subtypes associated with prognosis based on WNT gene family}

Nineteen WNT family genes were analyzed by univariate Cox regression analysis in UCEC, and the genes related to OS were identified. Clusterplus package was used for cluster analysis. The prognostic differences among different subgroups were further analyzed, and the survival curve was drawn with the survivalROC package. The clinical features such as clinical stage, tumor grade, tissue classification, TP53 mutation, cluster subgroup, age, and survival status were integrated, and a heatmap showing the correlation of the subtypes was drawn with the pheatmap package. Furthermore, principal component analysis (PCA) was carried out to compare the transcriptional spectrum of expression between different subgroups, and 
the ggplot2 package was used for visualization. The differentially expressed genes (DEGs) between subtypes were analyzed by the Limma package. The functional enrichment of gene pathways was analyzed by the gene set variation analysis (GSVA) package and visualized by the ggplot2 package. In addition, the hallmark dataset downloaded from the msigDB database was used for gene set enrichment analysis (GSEA) and further visualized by the enrichplot package.

\section{The construction of a multigene prognostic model}

The TCGA-UCEC cohort was divided into a training set and a validation set in a ratio of 1:1. In the training set (training set $=272$, validation set $=272$ ), 19 genes were analyzed by univariate Cox analysis using the survival package $(p<0.01)$. Using the glmnet package, Lasso cox analysis was further conducted to compress the number of genes in the risk model. Lasso regression analysis [13] is a compression estimate; it helps to obtain a more refined model by constructing a penalty function, compresses some regression coefficients by forcing the sum of the absolute values of the coefficients to be less than a fixed value, and sets some regression coefficients to zero. In this study, 10 cross-validation methods were used to construct the model, and the confidence interval (CI) of each $\lambda$ was analyzed.

\section{The construction of a nomogram using risk score and clinical features}

Nomograms [14] are based on a multifactor regression analysis approach, which integrates several predictive indicators and then draws a line segment with a scale on the same plane in a certain proportion; thus, nomograms can be used to express the relationship among the variables in a prediction model. A nomogram can individually calculate the survival rate of patients with specific tumors. The hazard ratio (HR), 95\% CI of the HR, and the $p$ value of the risk score were analyzed by univariate and multivariate Cox regression analyses, and the nomogram was constructed with multiple predictive variables.

\section{Immunohistochemical analysis of WNT2 and WNT10A in patients with UCEC}

We selected paraffin specimens resected from 75 patients with UCEC at Shengjing Hospital affiliated to China Medical University from 2007 to 2013. All patients were informed about the trial and signed an informed consent form, and the last follow-up date was July 20, 2020. Paraffin specimens of each UCEC tissue were fixed in $10 \%$ formalin and processed into sections at a thickness of $5 \mu \mathrm{m}$. Rabbit anti-human WNT2 polyclonal antibody was purchased from Solarbio (Beijing, China). Rabbit antihuman WNT10A polyclonal antibody was purchased from Proteintech (Wuhan, China). WNT2 and WNT10A expression was detected by the streptavidin-peroxidase method (SP). WNT2-positive paraffin sections of rat brain and WNT10A-positive paraffin sections of esophageal carcinoma served as positive controls. Phosphatebuffered saline (PBS) served as a negative control. The concentration of WNT2 polyclonal antibody working solution was 1:50 and that of WNT10A polyclonal antibody working solution was 1:100. The staining was carried out according to the SP kit instructions.

\section{Determination of the results of immunohistochemistry}

The staining of brown granules in cell membrane and cytoplasm was regarded as positive. Stained cells were classified based on their color intensity using the following score system: non-stained, light yellow, brownish yellow, and dark brown, which were recorded as 0, 1, 2 and 3 points, respectively. Five fields were randomly selected from each slice under a 400-fold optical microscope to observe the score, and the average value was taken as the percentage of stained cells: $<5 \%$ is $0,5-25 \%$ is $1,26-50 \%$ is $2,51-75 \%$ is 3 , and $>75 \%$ is 4 . The two items were multiplied to obtain the final score. The positive cell count and background evaluation were performed by two senior pathologists who were blinded to the patient data. Any objections were judged by a third pathologist. The immunohistochemical scores for WNT2 and WNT10A of each patient were substituted into the risk score formula, and the patients of the Shengjing cohort were divided into high-risk and low-risk groups according to the median risk score.

\section{Statistical analysis}

The Chi-square test and Fisher's exact probability test were used for the counting of data, and the Student's $t$-test was used for comparisons between the two groups. Analysis of variance (ANOVA), Kaplan-Meier analysis, and the log-rank test were used to analyze the survival curve, and univariate and multivariate Cox regression models were used to analyze the prognostic risk factors $(p<0.05)$. All statistical analyses were performed using $\mathrm{R}$ software (version 3.6.1).

\section{Results}

\section{Correlation of the WNT gene family and its expression with prognostic value in pan-cancer}

STRING database analysis showed the following values: number of nodes $=19$, number of edges $=171$, average node degree $=18$, protein-protein interaction $(\mathrm{PPI})$ enrichment $p<1.0 \mathrm{e}-16$. These results indicated that there was a strong interaction among the WNT family genes (Fig. 1A). The results of correlation analysis showed that there was a significant negative correlation 


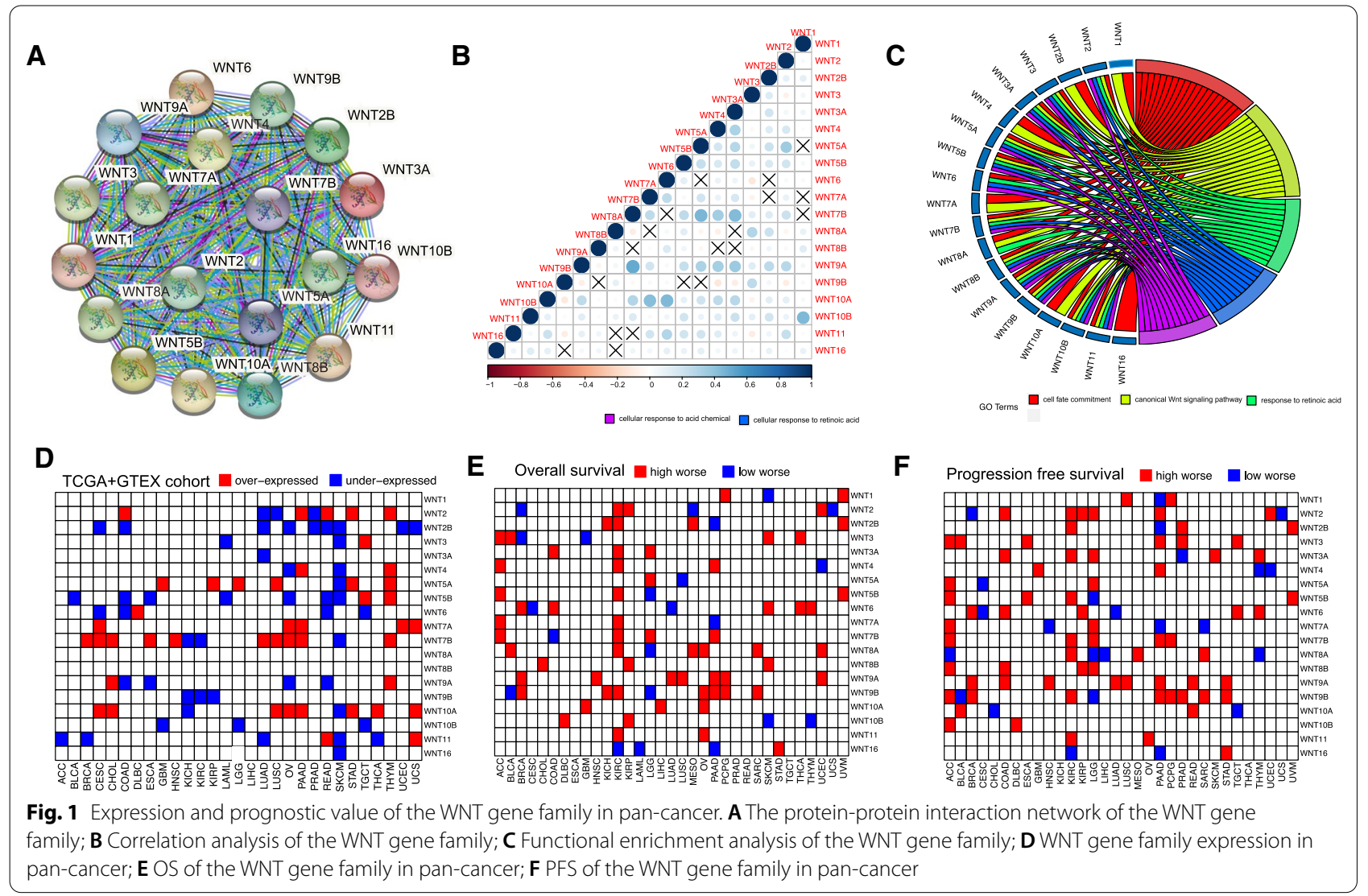

among most genes (Fig. 1B). Furthermore, GO functional enrichment analysis showed that most of the genes were enriched in cell fate commitment, the canonical WNT signaling pathway, and the response to the retinoic acid functional region (Fig. 1C). Differential expression analysis of the WNT gene family in pan-cancer showed that WNT2B was expressed at low levels in most tumors, WNT5A, WNT10A and WNT7B were highly expressed in most tumors, all WNT family genes were expressed at low levels in skin cutaneous melanoma (SKCM), and all WNT family genes were highly expressed in thymoma (THYM) (Fig. 1D). The relation between WNT family genes expression and OS or PFS in pan-cancer were further analyzed, and the results showed that most WNT genes were highly expressed in kidney renal clear cell carcinoma (KIRC) and adrenocortical carcinoma (ACC), suggesting poor prognosis. WNT9A, WNT8B, and WNT3A were highly expressed in most tumors with poor prognosis (Fig. 1E, F).

Correlation among the WNT gene family, immune subtype, and TP53 mutation

In previous studies, immune-genomic analysis was performed on more than 10,000 tumor samples from 33 cancer types from TCGA. In cross-tumor studies, six immune subtypes including $\mathrm{C} 1$ (wound healing), $\mathrm{C} 2$ (INF-r-dominant), C3 (inflammation), C4 (lymphocyte depletion), C5 (immunologically silent), and C6 (TGF$\beta$-dominant) were identified [15]. In view of the immunological silence of the C5 subtype, we analyzed the other five immune subtypes. The results showed that the expression of WNT2, WNT3, WNT3A, WNT4, WNT5A, WNT7A, WNT9A, WNT10A, WNT10B, and WNT16 were significantly different in different subtypes (Fig. 2A). The TP53 tumor suppressor gene is the most commonly altered gene in human tumors. Studies have shown that p53 mutations are widespread in UCEC, especially in type II UCEC, and TP53 expression increases gradually with disease progression [1618]. It has been suggested that TP53 mutation may be an independent prognostic factor for endometrial cancer. Therefore, the correlation between the WNT gene family and TP53 mutation was analyzed. The results showed that the WNT2, WNT3, WNT3A, WNT5A, WNT7A, WNT8B, and WNT10A genes were significantly associated with TP53 mutation (Fig. 2B). These findings suggest that TP53 mutation may be involved in malignant tumor progression via activation of the $\mathrm{WNT} / \beta$-catenin signaling pathway. 


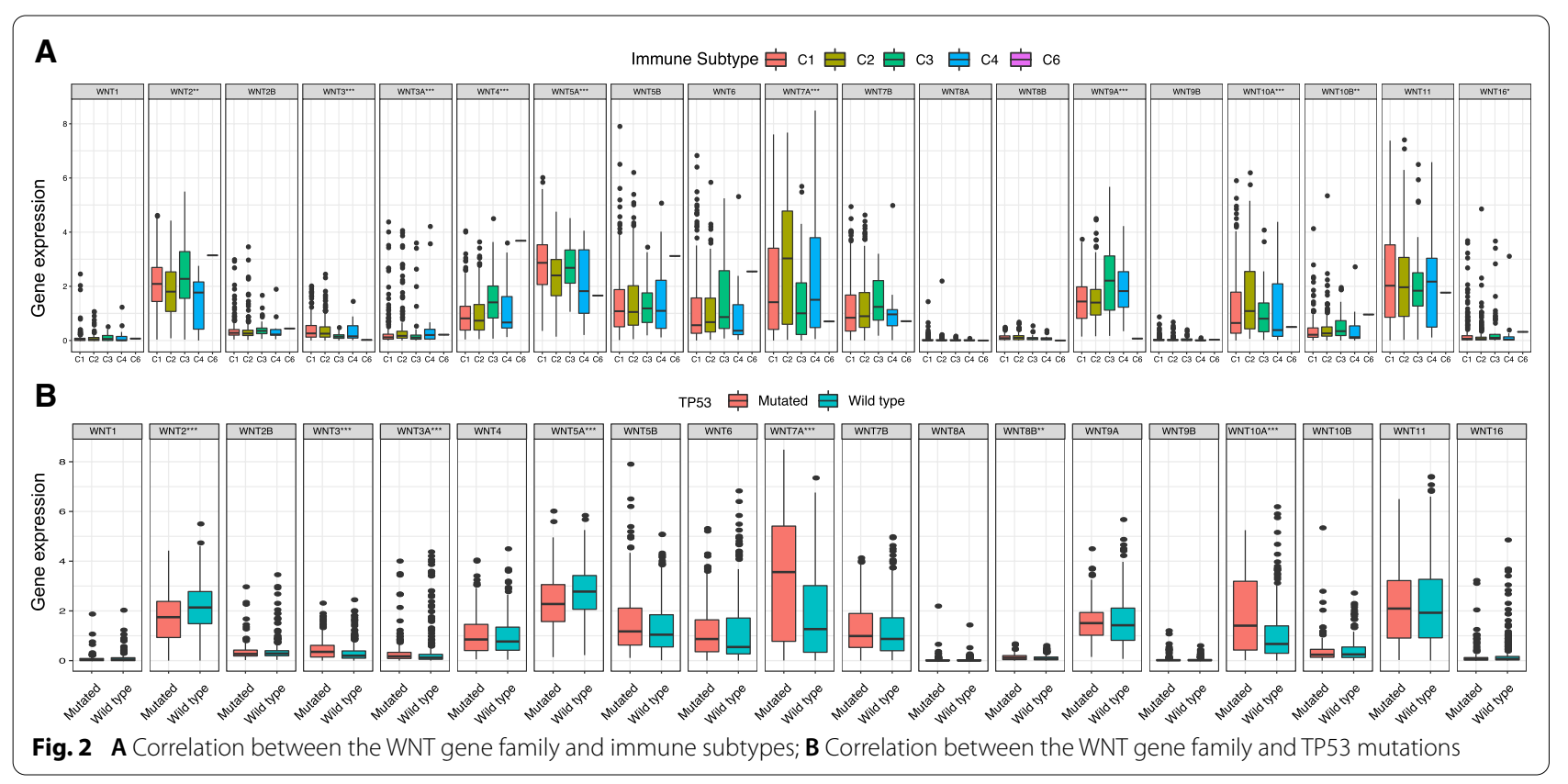

\section{Relation between the WNT gene family} and clinicopathological parameters of UCEC

Further analysis of the correlation between the WNT gene family and clinicopathological parameters of UCEC showed that the WNT1, WNT3, WNT7A, WNT7B, WNT8B, and WNT10A genes were expressed at significantly different levels at different tumor stages (Fig. 3A). There were significant differences in the expression levels of WNT2, WNT3, WNT3A, WNT4, WNT5A, WNT6, WNT7A, WNT8B, and WNT10B among different tumor grades (Fig. 3B). The expression of the WNT1, WNT2, WNT3A, WNT5A, WNT7A, WNT9A, and WNT10A genes was significantly different in different age groups (Fig. 3C). There were significant differences in WNT2, WNT3, WNT3A, WNT4, WNT5B, WNT6, WNT7A, WNT7B, WNT8B, WNT9A, WNT10A, and WNT11 expression among different histological types (Fig. 3D).

\section{Molecular subtype and functional enrichment analysis based on WNT family genes}

Four genes including WNT2, WNT5B, WNT7A, and WNT10A were identified by univariate Cox regression analysis in all TCGA datasets, and cluster analysis was performed using the Clusterplus package. The results showed that WNT2, WNT5B, WNT7A, and WNT10A were related to the prognosis of UCEC. In the TCGA dataset, the cumulative distribution function (CDF) curve of the WNT family genes showed that $\mathrm{k}=3$ seems to be an appropriate choice for cluster, but the clustering effect was more stable when $\mathrm{k}=2$
(Fig. 4A-C). Patients with UCEC were therefore divided into $\mathrm{C} 1$ and $\mathrm{C} 2$ subtypes. The DEGs between the $\mathrm{C} 1$ and $\mathrm{C} 2$ subtypes were analyzed using the Limma package [12], and a heatmap was drawn (Fig. 4D). Survival analysis showed that there was a significant difference between the $\mathrm{C} 1$ and $\mathrm{C} 2$ subtypes. The prognosis of the C1 subtype was significantly better than that of the $\mathrm{C} 2$ subtype (Fig. 4E). The functional enrichment of DEGs between the two subtypes was analyzed. The results of biological process enrichment are shown in Fig. 4F, which were mainly enriched in BIOLOGICAL_ADHESION, POSITIVE_REGULATION_OF_SIGNALING, APOPTOTIC_SIGNALING_PATHWAY, and REGULATION_OF_CELL_POPULATION_PROFLIFERATION. The results of Kyoto Encyclopedia of Genes and Genomes (KEGG) pathway enrichment analysis are shown in Fig. 4G; these were mainly enriched in ECM RECEPTOR_INTERACTION, ERBB_ SIGNALING_ PATHWAY, P53_SIGNALING_PATHWAY, and GNRH SIGNALING_PATHWAY. In addition, the results of GSEA analysis using the hallmarker gene set showed that the DEGs were enriched in G2M checkpoints, IL6_JAK_ STAT3 pathway, and KRAS pathway (Fig. 4H).

\section{Construction and validation of a 2-gene signature}

We sought to further explore the prognostic role of WNT family genes in UCEC. Univariate Cox analysis of 19 genes using the survival package showed that WNT2, WNT7A, WNT10A, and WNT16 were significantly correlated with OS $(p<0.05)$ (Fig. 5A). The change trajectory of independent variables showed that 

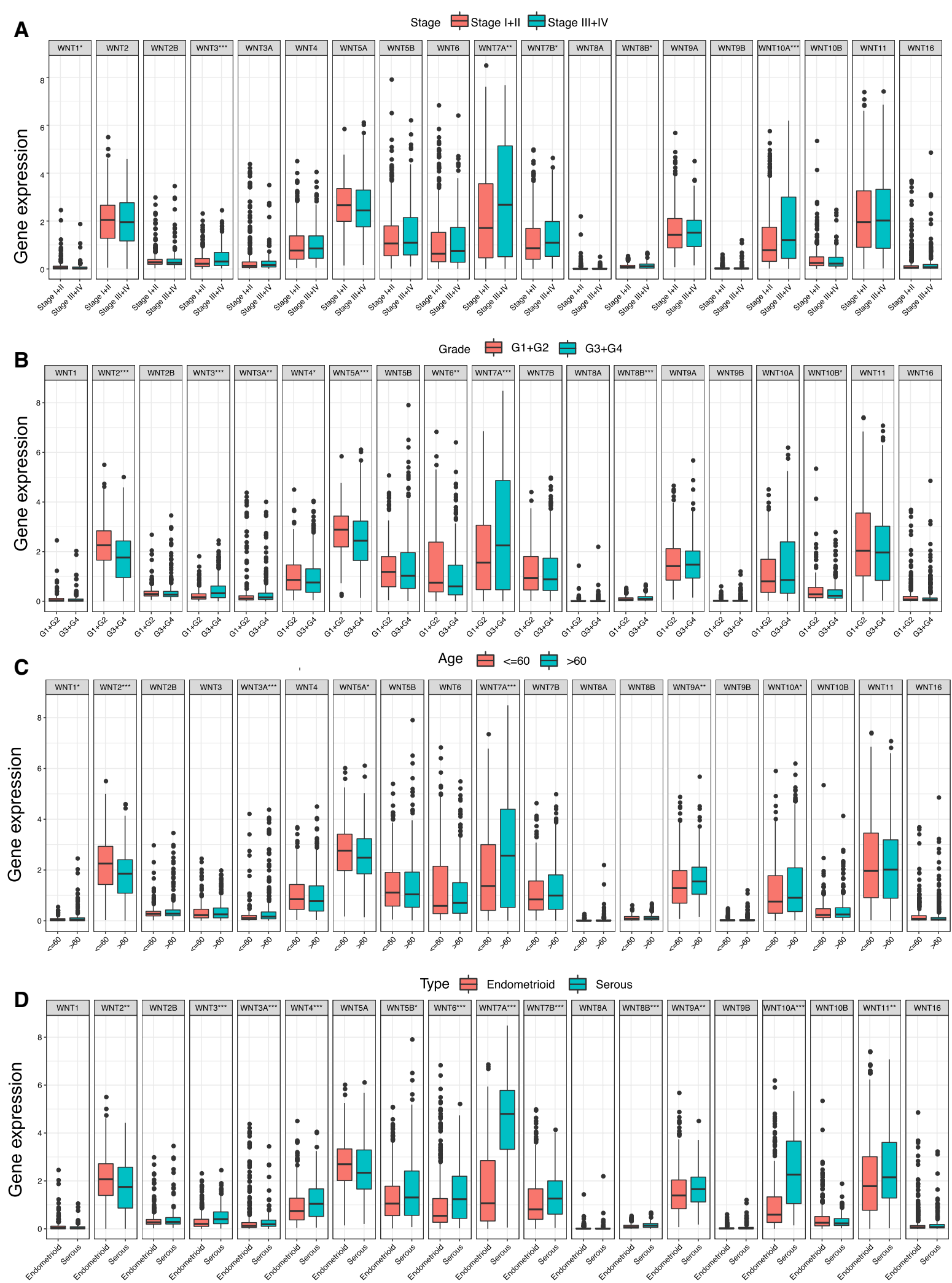

Fig. 3 Relationship between the WNT gene family and clinicopathological parameters of UCEC.A Correlation between expression of WNT family genes and FIGO stage; B Correlation between expression of WNT family genes and grade; C Correlation between expression of WNT family genes and age; D Correlation between expression of WNT family genes and tissue subtypes 


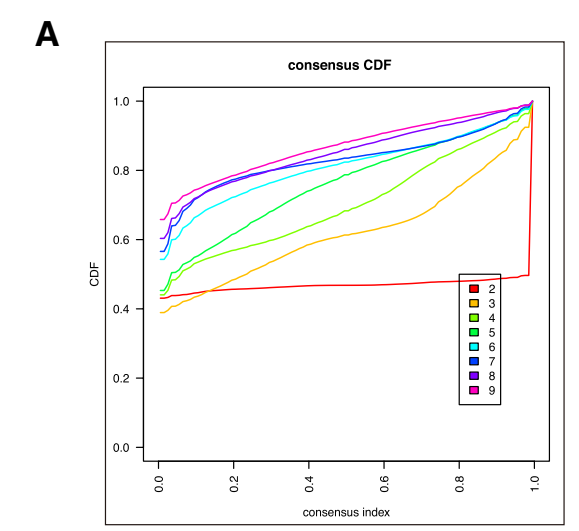

B

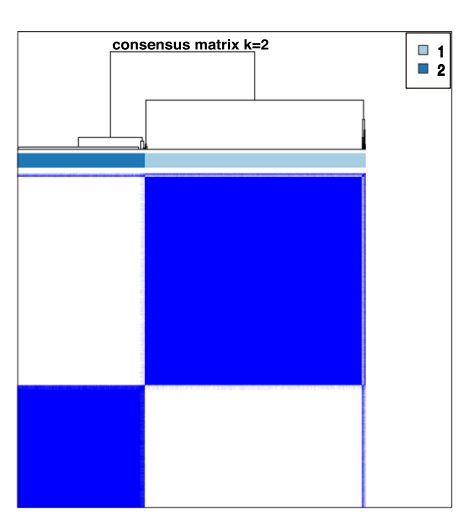

C

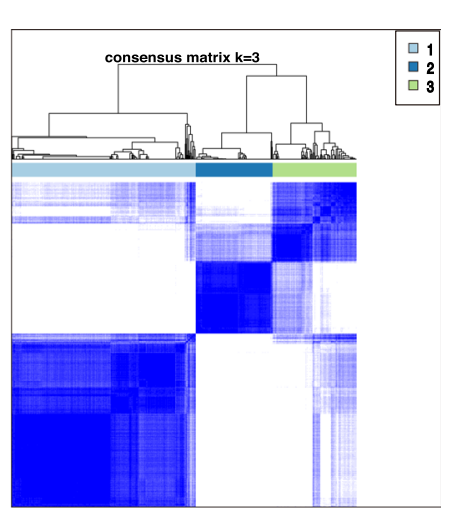

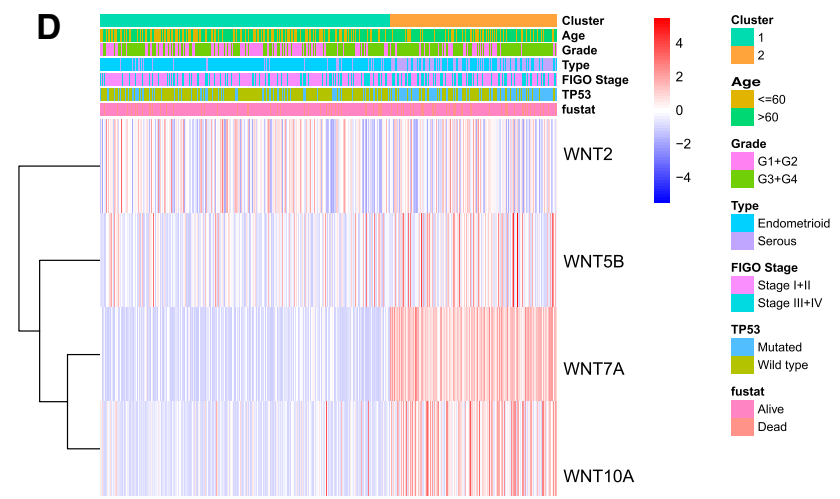

$\mathbf{E}$
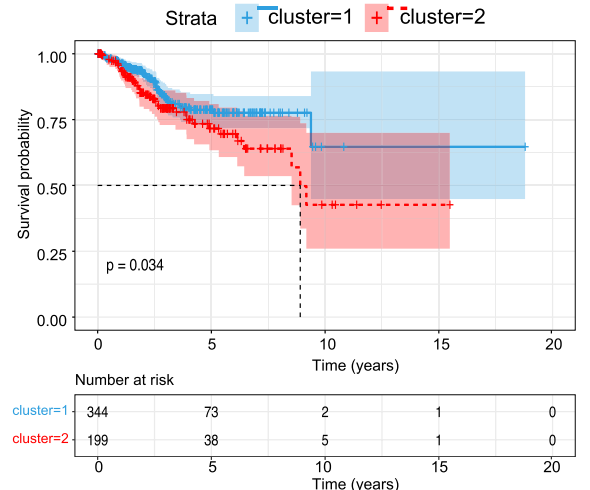

H

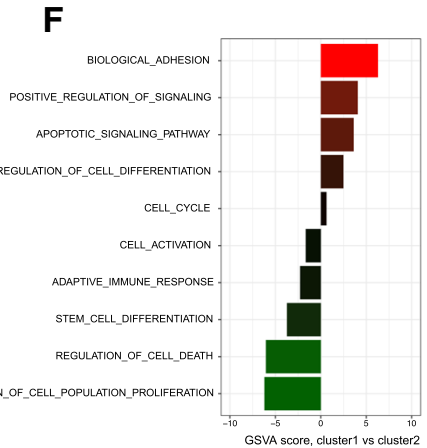

G
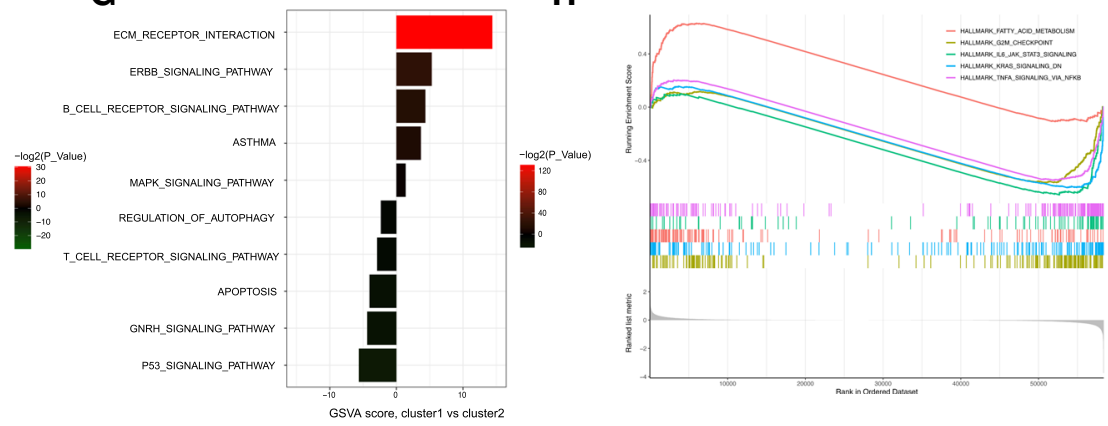

Fig. 4 Molecular typing of UCEC based on the WNT gene family.A Cumulative distribution function (CDF) curve; different colors represent different cluster numbers. The horizontal axis represents the consensus index, the vertical axis represents the CDF, and a bigger AUC indicates better clustering; $\mathbf{B}$ Heatmap of sample clustering at consensus $\mathrm{k}=2 ; \mathbf{C}$ Heatmap of sample clustering at consensus $\mathrm{k}=3$; $\mathbf{D}$ Gene expression heatmap of significantly prognostic genes in two subtypes. Red represents high expression and blue represents low expression; $\mathbf{E}$ Survival curve between different cluster groups; $\mathbf{F}$ Enrichment histogram of DEGs between cluster1 and cluster2 in biological process enrichment; $\mathbf{G}$ Enrichment histogram of DEGs between cluster1 and cluster2 in KEGG; $\mathbf{H}$ Enrichment analysis of DEGs in a hallmark gene set

the number of independent variable coefficients tending towards zero gradually increased with the gradual increase of lambda (Fig. 5C). The 10-fold cross-validation method was used to build the model, and the CI under each lambda was analyzed. The results showed that when $\log ($ lambda $)=-6.3$, the model is optimal; we therefore chose the four genes as target genes when log (lambda) $=-6.3$ (Fig. 5B). Multivariate Cox regression analysis showed that the WNT2 and WNT10A genes were still significantly associated with prognosis. The risk model of the two genes is as follows: risk score $=$ -0.333 "WNT2 + 0.337"WNT10A.

The risk score of each patient was calculated according to this formula. The results showed that with an increasing risk score, the age of the patients was older, the tumor stage was later, the tissue grade was higher, there were 
A

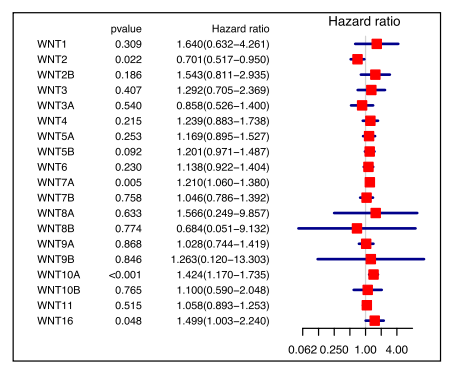

B

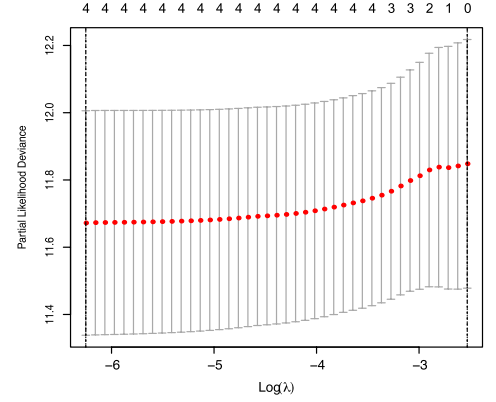

D Patients (increasing risk socre)
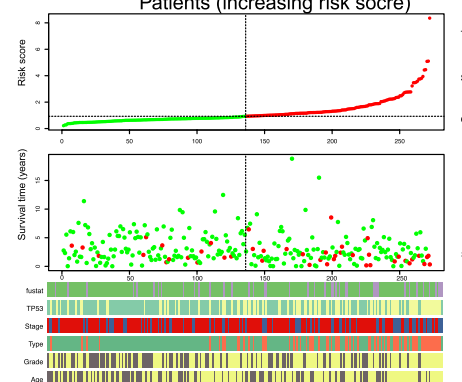

G

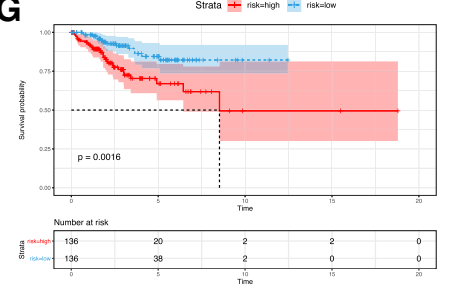

E TCGA entire cohort
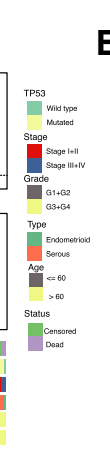

H

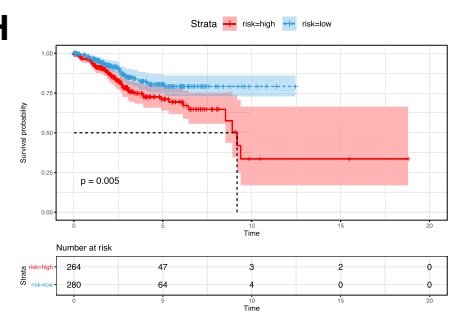

C

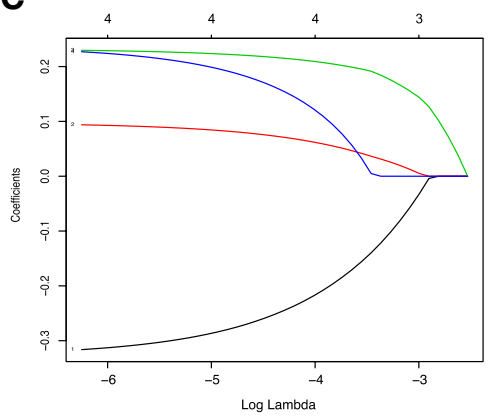

$\mathbf{F}$

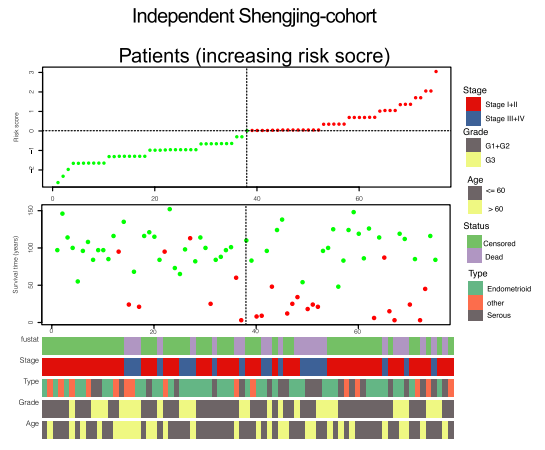

I
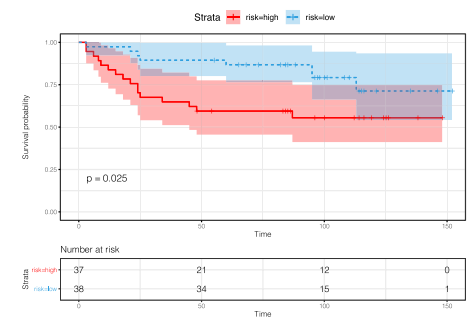

Fig. 5 Construction and validation of the 2-gene signature based on the WNT gene family.A Univariate cox analysis of WNT family genes in the TCGA-UCEC cohort training set; $\mathbf{B}$ The confidence interval under each lambda; $\mathbf{C}$ The changing trajectory of each independent variable. The horizontal axis represents the log value of the independent variable lambda, and the vertical axis represents the coefficient of the independent variable; D Risk score, survival time, survival status, and 2-gene signature expression in the TCGA training set; E Risk score, survival time, survival status, and 2-gene signature expression in all TCGA datasets; F Risk score, survival time, survival status, and 2-gene signature expression in the Shengjing cohort; G The KM survival curve distribution of the 2-gene signature in the TCGA training set; $\mathbf{H}$. he KM survival curve distribution of the 2-gene signature in all TCGA datasets; IThe KM survival curve distribution of the 2-gene signature in the Shengjing cohort

more TP53 mutations, and the survival status was worse (Fig. 5D-E). Patients were divided into high-risk and low-risk groups according to the median risk score value. Survival analysis showed that there were significant differences between the high-risk and low-risk groups, both in the training set and in all datasets (Fig. 5G, H).

The Shengjing UCEC cohort was used to further verify the prognostic ability of the risk score in the real world. Firstly, immunohistochemistry was used to evaluate WNT2 and WNT10A expression in patients with UCEC, and the representative weakly and strongly stained images were selected, as shown in Fig. 6. The risk score of each patient was calculated according to the formula and the risk score coefficient in the training set. The patients were divided into high-risk and low-risk groups according to the median risk score value. The results of survival analysis showed that there were still differences in survival outcomes between the high-risk and low risk group in an external independent cohort (Fig. 5F).

\section{Correlation between risk score and clinical subgroups}

The correlation between clinical subgroups and risk score was further analyzed. The results showed that the risk score of patients with age $>60$, stage III + IV cancer, serous type, mutated type was significantly higher than that of other patient groups, indicating that our signature 


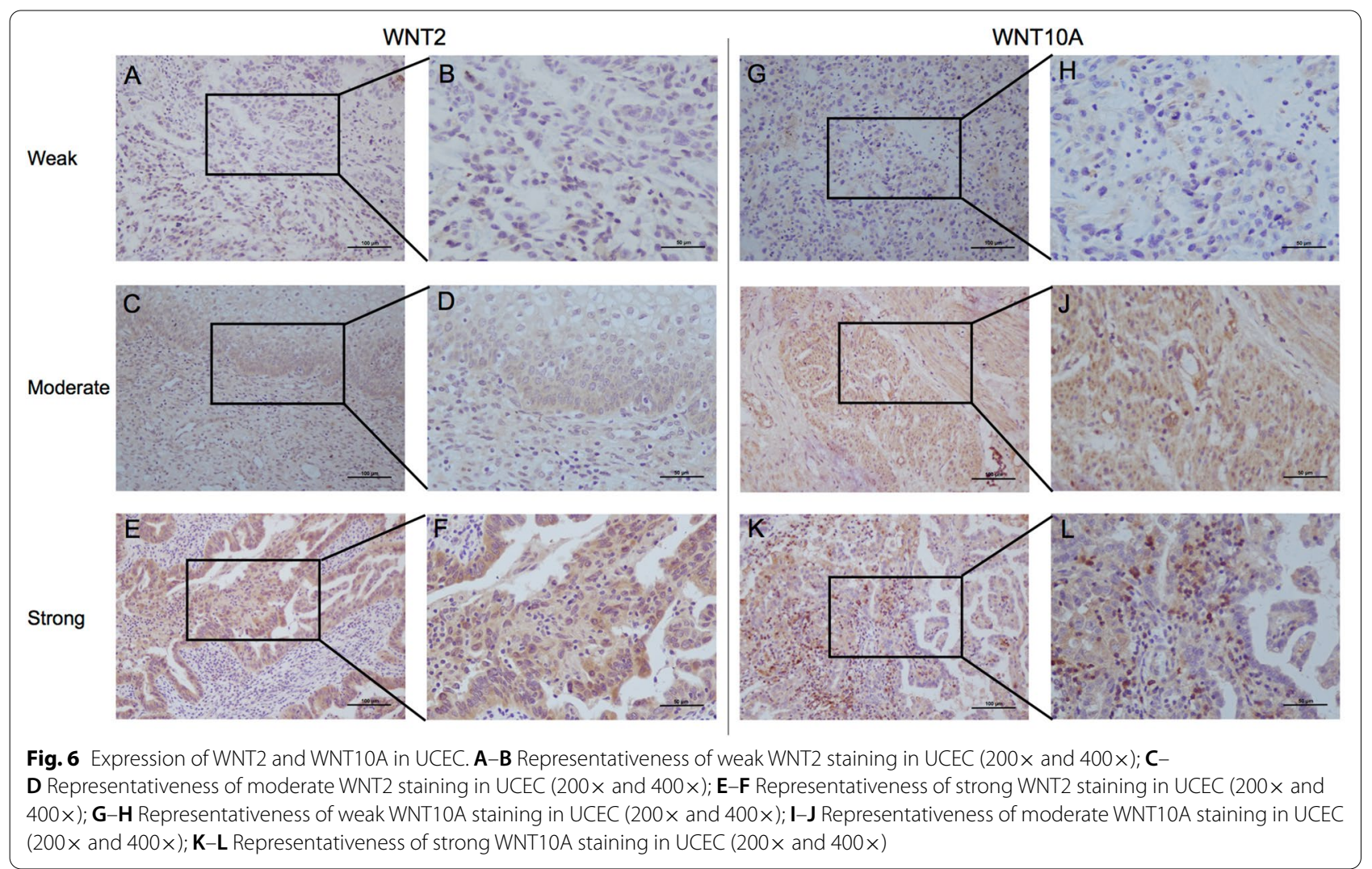

can facilitate subgroup diagnosis according to different clinical features (Fig. 7).

\section{Nomogram and its clinical diagnostic ability}

We further analyzed the relationship between risk score and other variables and the prognosis of patients with UCEC. Forest maps can simply and intuitively show the statistical results of different factors, as shown in(Fig. 8A, B. The risk score $(\mathrm{HR}=1.362, p=0.026)$, FIGO stage $(\mathrm{HR}=2.539, p=0.006)$, and grade $(\mathrm{HR}=3.274, p=0.035)$ were significantly correlated with survival and were independent risk factors for the prognosis of patients with UCEC. A nomogram was constructed with the stage, grade, and risk score. As observed from the results of the model, the risk score has the greatest influence on predicting the survival outcome, indicating that the risk model based on the WNT2 and WNT10A genes can better predict the prognosis of endometrial cancer (Fig. 8C). The performance of the 3- and 5-year nomograms can be displayed using a calibration plot. It showed that the nomogram performed well in predicting the prognosis of UCEC (Fig. 8D). The 3-5-year area under the curve (AUC) of the nomogram was also the largest when compared with the other clinical variables (Fig. 8E, F). Decision curve analysis (DCA) was used to evaluate the clinical effectiveness of the model, and the results showed that the nomogram had the best net benefit for predicting patient survival (Fig. 8G, H).

These results showed that compared with the nomogram constructed using a single clinical factor, the nomogram comprising risk score and significant variables constructed using a combined model is the best for predicting UCEC patients' survival. This model may therefore be helpful for clinical decision-making and personalized treatment.

\section{Discussion}

According to the traditional classification, UCEC can be divided into type I and type II, based on different origins, pathogenesis, and genetic characteristics [19]. Type I UCEC is estrogen-dependent and usually has a good prognosis. At the molecular level, type I endometrial cancer is associated with mutations in genes such as PTEN, KRAS, ARID1A, PIK3CA, and CTNNB1 and microsatellite instability (MSI). Abnormal changes in the genes may result in abnormal PTEN-PIK3/AKT/mTOR signaling pathways, which can lead to tumor development [20]. Type II UCEC is non-estrogenic and has a poor prognosis. At the molecular level, type II endometrial cancer is characterized by p53 mutations and HER2 


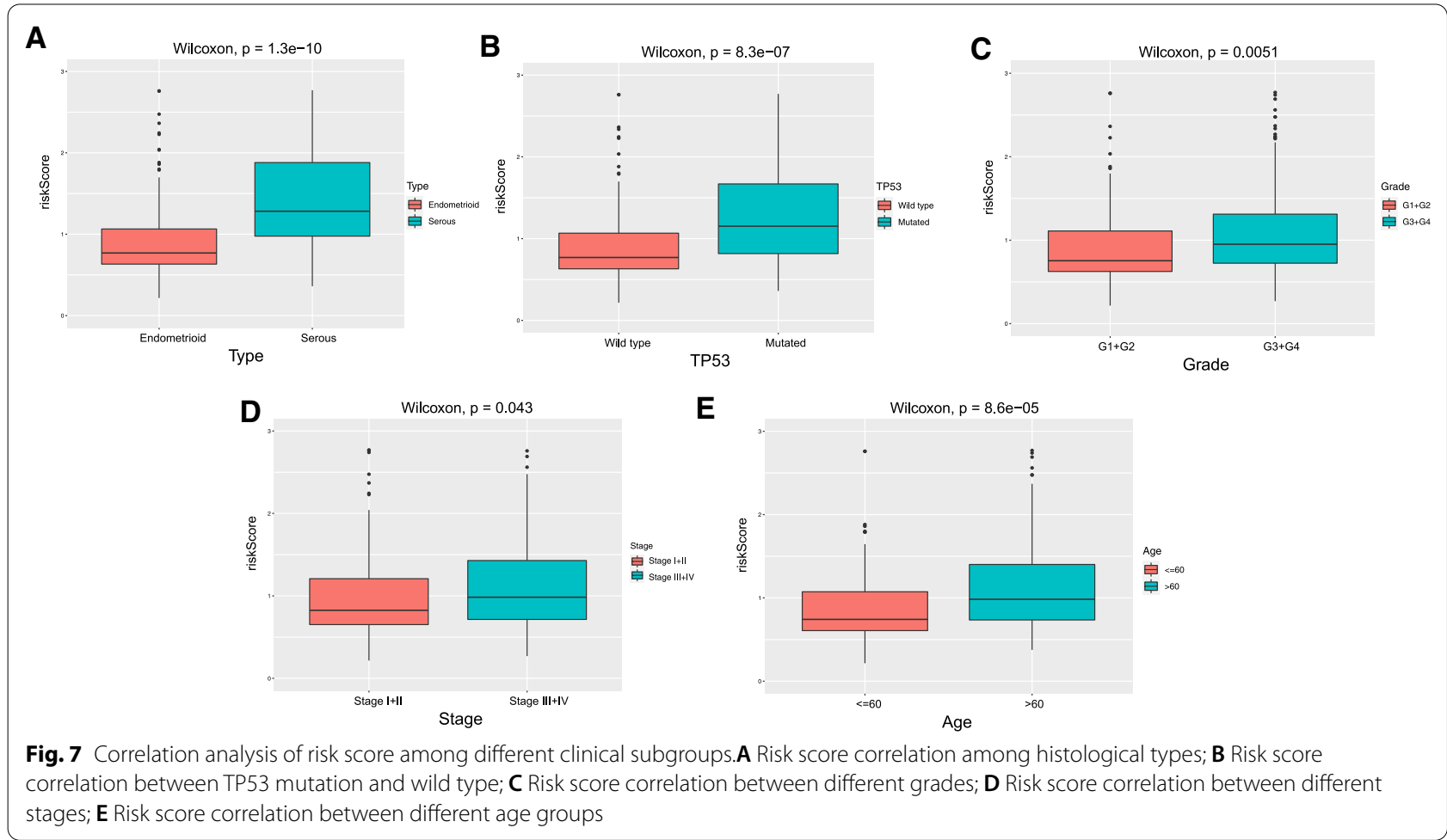

overexpression [21]. At present, clinicopathological features of UCEC such as pathological type and grade, FIGO stage, myometrial invasion, and lymphatic vascular invasion (LVSI) are used for risk stratification and diagnostic and therapeutic decision-making [22]. However, due to the heterogeneity of UCEC, several studies have focused on molecular changes that occur at the genetic level in UCEC $[23,24]$. Changes in the $\mathrm{WNT} / \beta$-catenin pathway are found in about $65 \%$ of the patients with UCEC [25]. The WNT/ $\beta$-catenin pathway is involved not only in the regulation of normal endometrium but also in endometrial hyperplasia and carcinogenesis [26]. As the promoter of the $\mathrm{WNT} / \beta$-catenin signaling pathway, the WNT gene family has attracted much attention. By analyzing the expression profiles and follow-up data of 19 molecules encoded by the WNT gene family in different types of tumors, the results showed that some WNT gene family genes can be used as prognostic indicators for patients. For example, WNT2 overexpression in colorectal cancer and hepatocellular carcinoma indicates poor prognosis [27, 28]. Additionally, WNT3A overexpression in esophageal squamous cell carcinoma and hepatocellular carcinoma indicates poor prognosis $[29,30]$.

Despite these findings, few studies have been conducted on UCEC thus far. Based on existing evidence, we speculated that the WNT gene family also plays an important role in predicting prognosis and risk stratification of UCEC. Since multiple factors may influence the expression of a single gene, it is not sufficient to independently predict prognosis in patients with UCEC. Compared with a single biomarker, the combined model constructed with multiple related genes is more accurate in predicting prognosis and is of great significance for individual diagnosis and treatment and for predicting UCEC patient prognosis [11, 31, 32]. Therefore, this study comprehensively evaluated the prognostic value of the WNT gene family in UCEC for the first time.

By analyzing the expression and prognosis of WNT family genes in pan-cancer, we found that WNT9A was overexpressed in most tumors and indicated poor prognosis. This is consistent with previous research results [33]. In UCEC, both PFS and OS were shortened in patients with high WNT2 expression, indicating poor prognosis. Studies have shown that the $\mathrm{WNT} / \beta$-catenin signaling pathway is involved in tumor cell immune escape. In a teratoma model, increased WNT expression was related to impaired immune cell recruitment and decreased $\mathrm{T}$ cell and $\mathrm{B}$ cell infiltration, suggesting that the immune surveillance function was impaired [34]. Further analysis of the correlation between WNT family genes and immune subtypes showed that there were significant differences in the expression levels of WNT2, WNT3, WNT3A, WNT4, WNT5A, WNT7A, WNT9A, WNT10A, WNT10B, and WNT16 among different 
A

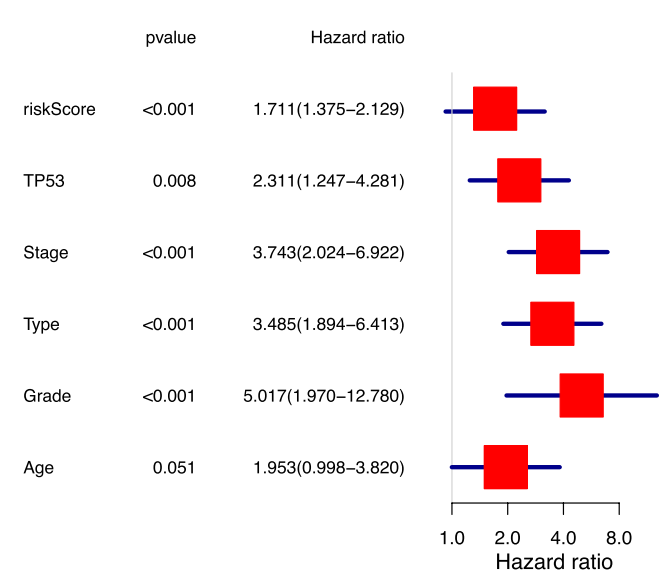

C

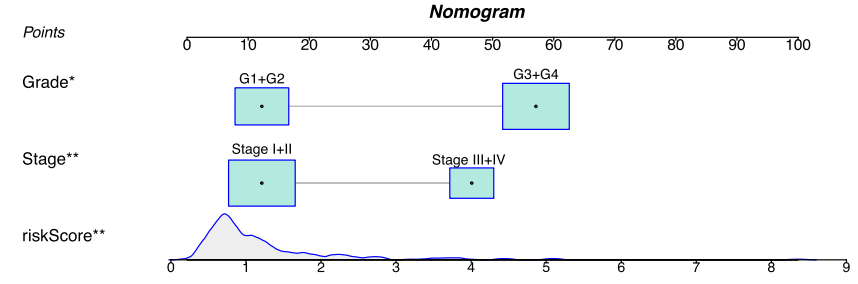

Total points

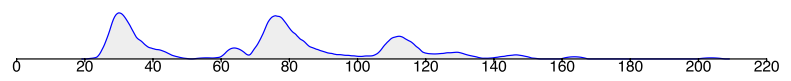

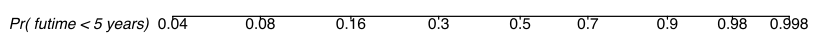

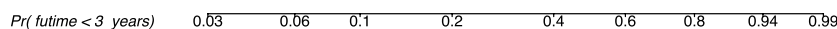

B

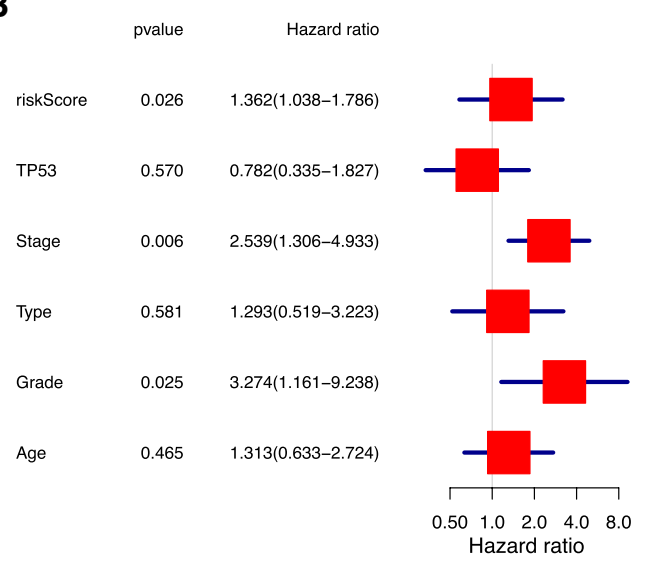

D

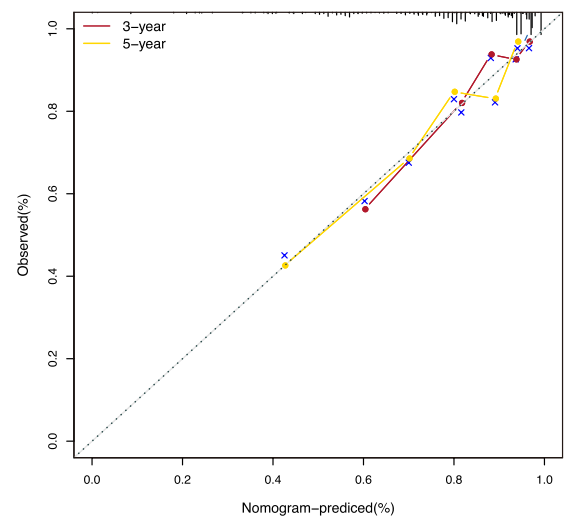

$\mathbf{E}$

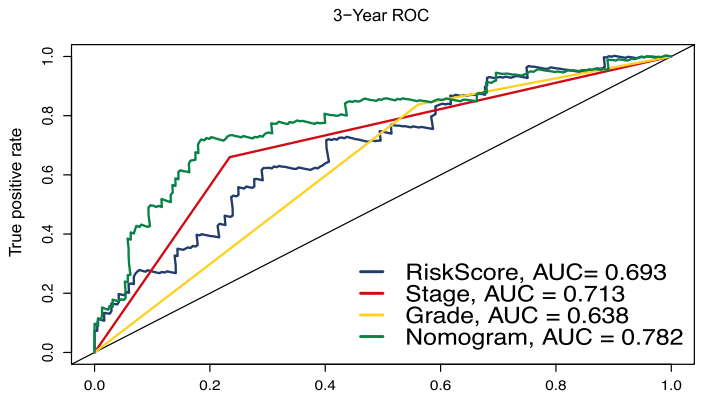

G

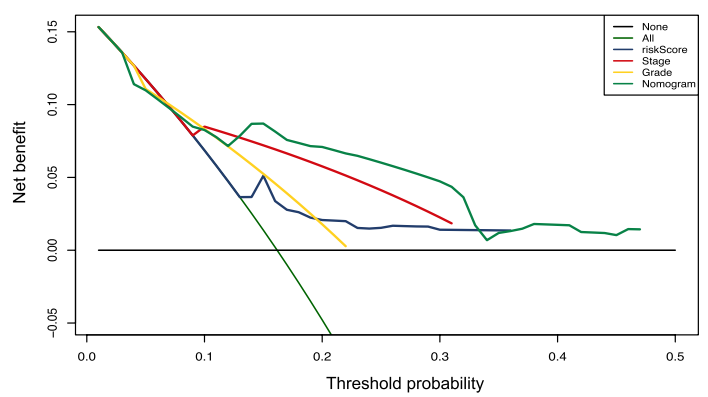

$\mathbf{F}$

5 -Year ROC

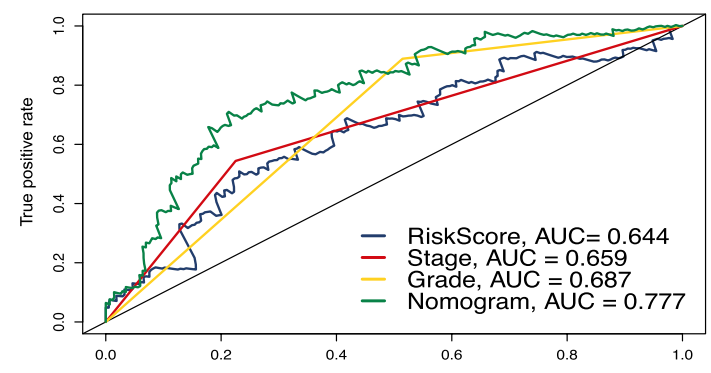

H

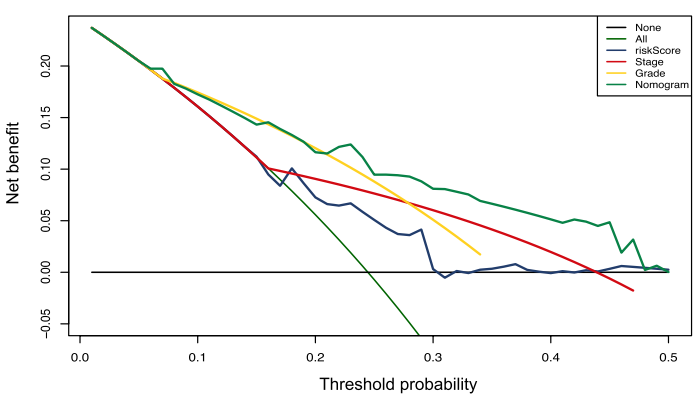

Fig. 8 Clinical diagnostic value of the 2-gene signature. A Forest map of univariate Cox analysis; B Forest map of multivariate Cox analysis; C Nomogram for predicting the 3- and 5-year OS of patients; D The 3- and 5-year calibration curves of the nomogram; $\mathbf{E}$ The 3-year ROC of the nomogram; F The 5-year ROC of the nomogram; G The 3-year DCA curves of the nomogram; $\mathbf{H}$ The 5-year DCA curves of the nomogram 
immune subtypes. Studies have shown that when WNT2 is knocked down, IL-8 expression is increased in epithelial cells [35]. WNT5a has dual effects on the tumor microenvironment; it can activate the autocrine ROR1/ Akt/P65 pathway and promote immune cell inflammation and chemotaxis. WNT5a can also specifically activate the TLR/MyD88/p50 pathway in bone marrow monocytes and promote the synthesis of anti-inflammatory cytokines such as IL-10 and the tolerance phenotype, thus forming an immunosuppressive tumor microenvironment [36]. Although the mechanism of the WNT family genes involved in immune regulation is not clear, our results suggest that WNT family genes may be used as important markers to distinguish immune subtypes.

Further analysis of the correlation between the WNT gene family and the clinicopathological parameters of UCEC showed that WNT7A was significantly different in different clinical stages, tumor grades, age groups, and histological types. Some studies have shown that WNT7A is overexpressed in UCEC and indicates a poor prognosis [37]. However, its mechanism in the occurrence and development of UCEC still needs to be explored further.

We used the TCGA-UCEC cohort to analyze 19 WNT genes by univariate and multivariate Cox regression analyses and Lasso regression analysis. The results showed that WNT2 and WNT10A were significantly correlated with prognosis. The risk score of the 2-gene signature was constructed, and the patients were divided into high-risk and low-risk groups according to the median risk score. Although previous studies have evaluated the prognostic value of the WNT gene family in prostate cancer [38] and hepatocellular carcinoma [28], these studies are only included in the TCGA cohort. The main ethnic groups in the TCGA cohort are black and white, and there is a lack of data regarding the Asians. This study is the first time that a real-world cohort has been applied for studying UCEC, and thus its prognosis prediction is more reliable. Furthermore, the meaningful clinical features of multivariate Cox analysis were combined with the risk score to construct a nomogram. The results of the calibration map and DCA curve show that our model has a good ability to predict UCEC patient prognosis. Compared with the other prognostic models of UCEC [39, 40], our model contains fewer genes and is more convenient to use in clinical practice.

Despite these benefits, our research still has some limitations. Our study is a retrospective study that still needs to be verified by a prospective study in the future. Secondly, although we have included real-world data for verification, the sample size is small, and further research with a larger sample size is needed in the future.

\section{Conclusions}

Two genes (WNT2 and WNT10A) significantly related to prognosis of UCEC were identified by comprehensively analyzing the prognostic value of the WNT family and risk score was constructed. Patients with UCEC can be divided into high-risk and low- risk groups according to the risk score, and the high-risk group has a poor prognosis. The 2-gene signature provides new avenues for prognosis prediction and clinical decision-making in UCEC.

\section{Abbreviations \\ UCEC: Uterine corpus endometrial carcinoma; OS: Overall survival; FIGO: International Federation of Obstetrics and Gynecology; NCCN: National Committee on Computer Network; TCGA: The Cancer Genome Atlas; MSI: Mutation microsatellite instability; NSMP: Non-specific molecular variation; GTEx: Genotype-Tissue Expression; PFS: Progression-free survival; GO: Gene Ontology; PCA: Principal component analysis; DEGs: Differentially expressed genes; GSEA: Gene set enrichment analysis; GSVA: Gene set variation analysis; Cl: Confidence interval; HR: Hazard ratio; SP: Streptavidin-peroxidase; PBS: Phosphate-buffered saline; ANOVA: Analysis of variance; PPI: Protein-protein interaction; THYM: Thymoma; SKCM: Skin Cutaneous Melanoma; KIRC: Kidney renal clear cell carcinoma; ACC: Adrenocortical carcinoma; CDF: cumulative distribution function; KEGG: Kyoto Encyclopedia of Genes and Genomes; AUC : Area under the curve.}

\section{Acknowledgements}

This study was approved by the Ethics Committee of China Medical University.

\section{Authors' contribution}

YH: conceptualization, methodology, data curation, visualization, formal analysis, writing-original draft preparation. MZ: methodology, formal analysis, visualization, writing-original draft preparation. DZ: data curation, visualization, writing-original draft preparation. RG: data curation, validation. OL: validation. SW: writing-review \& editing, project administration. BL: writing-review \& editing, supervision, project administration, funding acquisition. All authors read and approved the final manuscript.

\section{Funding}

This work was supported by Outstanding Scientific Fund of Shengjing Hospital (201804).

\section{Availability of data and materials}

The datasets used and analysed during the current study are available from the corresponding author on reasonable request.

\section{Declarations}

Ethics approval and consent to participate Not applicable.

\section{Consent for publication}

Not applicable.

\section{Competing interests}

The authors declare no conflicts of interest.

\section{Author details}

${ }^{1}$ Department of Gynecology and Obstetrics, Shengjing Hospital of China Medical University, Shenyang, China. ${ }^{2}$ Key Laboratory of Maternal-Fetal Medicine of Liaoning Province, Benxi, China. ${ }^{3}$ Key Laboratory of Obstetrics and Gynecology of Higher Education of Liaoning Province, Benxi, China. ${ }^{4}$ Department of Obstetrics and Gynecology, University Hospital, LMU Munich, Marchioninistr. 15, 81377 Munich, Germany. ${ }^{5}$ Present Address: 4th Gynecological Ward, Department of Obstetrics and Gynecology, Shengjing Hospital 
of China Medical University, 36 Sanhao Street, Liaoning 110004 Shenyang, People's Republic of China.

Received: 23 July 2021 Accepted: 15 September 2021 Published online: 26 September 2021

\section{References}

1. Global Burden of Disease, Cancer C, Fitzmaurice C, Akinyemiju TF, Al Lami FH, Alam T, Alizadeh-Navaei R, et al. Global, regional, and national cancer incidence, mortality, years of life lost, years lived with disability, and disability-adjusted life-years for 29 cancer groups, 1990 to 2016: a systematic analysis for the Global Burden of Disease Study. JAMA Oncol. 2018:4:1553-68.

2. Siegel RL, Miller KD, Fuchs HE, Jemal A. Cancer statistics. CA Cancer J Clin. 2021;71:7-33.

3. Morice P, Leary A, Creutzberg C, Abu-Rustum N, Darai E. Endometrial cancer. Lancet. 2016;387:1094-108.

4. Bell DW, Ellenson LH. Molecular genetics of endometrial carcinoma. Annu Rev Pathol. 2019;14:339-67.

5. Akalay I, Tan TZ, Kumar P, Janji B, Mami-Chouaib F, Charpy C, et al. Targeting WNT1-inducible signaling pathway protein 2 alters human breast cancer cell susceptibility to specific lysis through regulation of KLF-4 and miR-7 expression. Oncogene. 2015;34:2261-71.

6. Dale TC. Signal transduction by the Wnt family of ligands. Biochem J. 1998;329(Pt 2):209-23.

7. Yong X, Tang B, Xiao YF, Xie R, Qin Y, Luo G, et al. Helicobacter pylori upregulates Nanog and Oct4 via Wnt/beta-catenin signaling pathway to promote cancer stem cell-like properties in human gastric cancer. Cancer Lett. 2016;374:292-303.

8. Monteleone E, Orecchia V, Corrieri P, Schiavone D, Avalle L, Moiso E, et al. SP1 and STAT3 functionally synergize to induce the RhoU small GTPase and a subclass of non-canonical WNT responsive genes correlating with poor prognosis in breast cancer. Cancers (Basel). 2019;11:101.

9. Zhang X, Gaspard JP, Chung DC. Regulation of vascular endothelial growth factor by the Wnt and K-ras pathways in colonic neoplasia. Cancer Res. 2001;61:6050-4.

10. Cheng Y, Li X, Dai Y, Dong Y, Yang $X$, Wang J. Identification of an immunerelated risk signature and nomogram predicting the overall survival in patients with endometrial cancer. J Gynecol Oncol. 2021;32:e30.

11. Zhang J, Wang Z, Zhao R, An L, Zhou X, Zhao Y, et al. An integrated autophagy-related gene signature predicts prognosis in human endometrial Cancer. BMC Cancer. 2020;20:1030.

12. Ritchie ME, Phipson B, Wu D, Hu Y, Law CW, Shi W, et al. limma powers differential expression analyses for RNA-sequencing and microarray studies. Nucleic Acids Res. 2015;43:e47.

13. Tibshirani R. Regression shrinkage and selection via the lasso: a retrospective. 1996; 58.

14. Balachandran VP, Gonen M, Smith JJ, Dematteo RP. Nomograms in oncology: more than meets the eye. Lancet Oncol. 2015;16:e173-80.

15. Thorsson V, Gibbs DL, Brown SD, Wolf D, Bortone DS, Ou Yang TH, et al. The immune landscape of cancer. Immunity. 2018;48:812-830.e14.

16. Bur ME, Perlman C, Edelmann L, Fey E, Rose PG. p53 expression in neoplasms of the uterine corpus. Am J Clin Pathol. 1992;98:81-7.

17. Jia L, Liu Y, Yi X, Miron A, Crum CP, Kong B, et al. Endometrial glandular dysplasia with frequent p53 gene mutation: a genetic evidence supporting its precancer nature for endometrial serous carcinoma. Clin Cancer Res. 2008;14:2263-9.

18. Catasus L, Gallardo A, Cuatrecasas M, Prat J. Concomitant PI3K-AKT and p53 alterations in endometrial carcinomas are associated with poor prognosis. Mod Pathol. 2009;22:522-9.

19. Bokhman JV. Two pathogenetic types of endometrial carcinoma. Gynecol Oncol. 1983;15:10-7.
20. Hussein YR, Soslow RA. Molecular insights into the classification of highgrade endometrial carcinoma. Pathology. 2018;50:151-61.

21. Carlson JW, Nastic D. High-grade endometrial carcinomas: classification with molecular insights. Surg Pathol Clin. 2019;12:343-62.

22. McAlpine JN, Temkin SM, Mackay HJ. Endometrial cancer: not your grandmother's cancer. Cancer. 2016;122:2787-98.

23. Urick ME, Bell DW. Clinical actionability of molecular targets in endometrial cancer. Nat Rev Cancer. 2019;19:510-21.

24. Talhouk A, McAlpine JN. New classification of endometrial cancers: the development and potential applications of genomic-based classification in research and clinical care. Gynecol Oncol Res Pract. 2016;3:14.

25. McMellen A, Woodruff ER, Corr BR, Bitler BG, Moroney MR. Wht signaling in gynecologic malignancies. Int J Mol Sci. 2020;21:4272.

26. Coopes A, Henry CE, Llamosas E, Ford CE. An update of Wnt signalling in endometrial cancer and its potential as a therapeutic target. Endocr Relat Cancer. 2018;25:647.

27. Kramer N, Schmollerl J, Unger C, Nivarthi H, Rudisch A, Unterleuthner D, et al. Autocrine WNT2 signaling in fibroblasts promotes colorectal cancer progression. Oncogene. 2017;36:5460-72.

28. Han Q, Wang X, Liao X, Han C, Yu T, Yang C, et al. Diagnostic and prognostic value of WNT family gene expression in hepatitis B virusrelated hepatocellular carcinoma. Oncol Rep. 2019:42:895-910.

29. Pan LH, Yao M, Cai Y, Gu JJ, Yang XL, Wang L, et al. Oncogenic Wnt3a expression as an estimable prognostic marker for hepatocellular carcinoma. World J Gastroenterol. 2016;22:3829-36.

30. Oguma J, Ozawa S, Kazuno A, Nitta M, Ninomiya Y, Kajiwara H. Wnt3a expression is associated with poor prognosis of esophageal squamous cell carcinoma. Oncol Lett. 2018;15:3100-8.

31. Liu J, Li S, Feng G, Meng H, Nie S, Sun R, et al. Nine glycolysis-related gene signature predicting the survival of patients with endometrial adenocarcinoma. Cancer Cell Int. 2020;20:183.

32. Jiang P, Sun W, Shen N, Huang X, Fu S. Identification of a metabolismrelated gene expression prognostic model in endometrial carcinoma patients. BMC Cancer. 2020;20:864.

33. Ali I, Medegan B, Braun DP. Wnt9A induction linked to suppression of human colorectal cancer cell proliferation. Int J Mol Sci. 2016;17:495.

34. Augustin I, Dewi DL, Hundshammer J, Rempel E, Brunk F, Boutros M. Immune cell recruitment in teratomas is impaired by increased Wnt secretion. Stem Cell Res. 2016;17:607-15.

35. Liu X, Lu R, Wu S, Zhang YG, Xia Y, Sartor RB, et al. Wnt2 inhibits enteric bacterial-induced inflammation in intestinal epithelial cells. Inflamm Bowel Dis. 2012;18:418-29.

36. Lopez-Bergami P, Barbero G. The emerging role of Wnt5a in the promotion of a pro-inflammatory and immunosuppressive tumor microenvironment. Cancer Metastasis Rev. 2020;39:933-52.

37. Liu Y, Meng F, Xu Y, Yang S, Xiao M, Chen X, et al. Overexpression of Wnt7a is associated with tumor progression and unfavorable prognosis in endometrial cancer. Int J Gynecol Cancer. 2013;23:304-11.

38. Hu M, Xie J, Liu Z, Wang X, Liu M, Wang J. Comprehensive analysis identifying Wnt ligands gene family for biochemical recurrence in prostate adenocarcinoma and construction of a nomogram. J Comput Biol. 2020;27:1656-67.

39. Cai L, Hu C, Yu S, Liu L, Zhao J, Zhao Y, et al. Identification of EMT-related gene signatures to predict the prognosis of patients with endometrial cancer. Front Genet. 2020;11:582274.

40. Liu J, Jiang P, Chen X, Shen Y, Cui G, Ma Z, et al. Construction of a nine DNA repair-related gene prognostic classifier to predict prognosis in patients with endometrial carcinoma. BMC Cancer. 2021;21:29.

\section{Publisher's Note}

Springer Nature remains neutral with regard to jurisdictional claims in published maps and institutional affiliations. 